\title{
A Note on the Formation and Structure of Iron Dendrites in a Magnesium Alloy
}

\author{
By Harry C. Burnett, Jr. and Herbert C. Vacher
}

\begin{abstract}
Metallographic examination of commercially pure magnesium that had been heated to $1,000^{\circ} \mathrm{C}$ in an iron crucible revealed iron dendrites. Further examination by the microradiographic method showed that the dendrites grew at uniform rates in the dodecahedral directions.
\end{abstract}

During a metallographic examination of commercially pure magnesium, several six-pointed, star-shaped inclusions were observed, see figure 1. This magnesium had been heated to $1,000^{\circ} \mathrm{C}$ in an. Armeo iron crucible and stirred with an iron. rod. The presence of similar inclusions in magnesium has been reported by Beck ${ }^{1}$ and by Fahrenhorst and Bulion, ${ }^{2}$ who concluded that they were iron dendrites. Fahrenhorst and Bulion, reported that the solubility of iron in liquid magnesium at the melting point $\left(650^{\circ} \mathrm{C}\right)$ was 0.025 percent, and at $1,200^{\circ} \mathrm{C}$ it was 0.84 percent. These values were substantially verified by Mitchell. ${ }^{3}$

Fahrenhorst and Bulion. discussed the manner of growth of iron dendrites but did not clearly state whether they grew in the cubic or the octahedral directions or a combination, of both. There are six cubic directions, and if the iron dendrites grew only in these directions the maximum number of branches in a plane passing through the nucleus would be four, and they would include angles of $90^{\circ}$. Likewise, there are eight ocrahedral directions and if the dendrites grew in these directions only, the maximum number of branches in a plane passing through the nucleus would also be four, but they would include angles of $70^{\circ}$ and $110^{\circ}$. The dendrite in figure 1, which is a plane section. through the nucleus, has six branches. It can be concluded that the growth of the dendrite was not in the cubic or octahedral directions alone.

\footnotetext{
A dolph Beck, The technology of magnesium and its alloys, p. 43 (1941).

${ }^{2}$ E. Fahrenhorst and W. Bulion, Metallography of magnesium and its alloys, p. 27 (1947).

${ }^{3}$ D. W. Mitchell, Solubility of iron in magnesium, Metals technology, Am. Inst. Min. Met. Engr. 15, No. 1 (Jan. 1948).
}

If iron dendrites grew in magnesium in both the cubic and octahedral directions simultaneously, the maximum number of branches in a plare passing through the nucleus would be six-two in. cubic directions and four in, octahedral directions. The angle between adjacent branches in the octahedral directions would be $70^{\circ}$, and the angle between adjacent cubic and octahedral directions is $55^{\circ}$. Starting with a cubic direction the angular spacing between, adjacent branches is $55^{\circ}$, $70^{\circ}, 55^{\circ}, 55^{\circ}, 70^{\circ}$, and $55^{\circ}$. In order to test this possibility, the angles between the more perfectly developed branches $A, B, C$, and $D$ in. figure 1 were measured as accurately as possible and were found to be $60^{\circ}$ between $A$ and $B, 62^{\circ}$ between, $B$ and $C$, and $58^{\circ}$ between $C$ and $D$. These results preclude the possibility that the iron dendrites grow in a combination of cubic and octahedral directions. Since the maximum number of branches in any one plane passing through the center of a dendrite that had grown in the dodecahedral directions would be six, and the angular spacing between, the branches would be $60^{\circ}$, it may be presumed that the branches of iron dendrites in magnesium grow in the twelve dodecahedral directions.

In order to obtain more complete data on the appearance of iron dendrites, microradiographs were made. A specimen, $3 / 8$ in. by $3 / 4$ in. having a thickness of $0.010 \mathrm{in}$. was prepared from the same material in. which was found the dendrite shown in figure 1. Copper radiation was used with a specimen-to-film distance of $28 \mathrm{~cm}$. The photomicroradiograph in figure 2 shows a cluster of dendrites. A study of these and others shows 
that there are certain characteristic details that indicate that the dendrites grew in twelve directions, that is, in the dodecahedral directions.

In interpreting the photomicroradiographs in figures 2,3 , a, 4, a, 5, a, and 6, a, it should be kept in mind that the images are silhouettes of threedimensional inclusions made by projecting them perpendicularly onto a flat photographic plate. In these photomicroradiographs it will be noted that certain branches are lighter than others, that the number of branches varies, and that the angles between the branches differ. To consider these variations systematically a model was made of a hypothetical dendrite that grew at the same rate in all twelve dodecahedral directions. It was then photographed using a long focal-length lens so that the photographs would simulate a photomicroradiograph, with the exception that variation in spoke intensity could not be produced. The positions of the model were such that the optical axis of the camera coincided with an octahedral axis, figure $3, \mathrm{~b}$; a dodecahedral axis, figure 4 , b; a cubic axis, figure 5 , b; and an icositetrahedral axis, figure $6, \mathrm{~b}$. The orientations of the spokes of the model (dodecahedral directions) in the photographs $3, \mathrm{~b}, 4, \mathrm{~b}, 5, \mathrm{~b}$, and 6 , b, are shown in the stereographic projections $3, \mathrm{c}, 4, \mathrm{c}, 5$, c, and 6 , c respectively. The plane of projection in each case corresponds to the photographic film. The photomicroradiographs were then inspected to

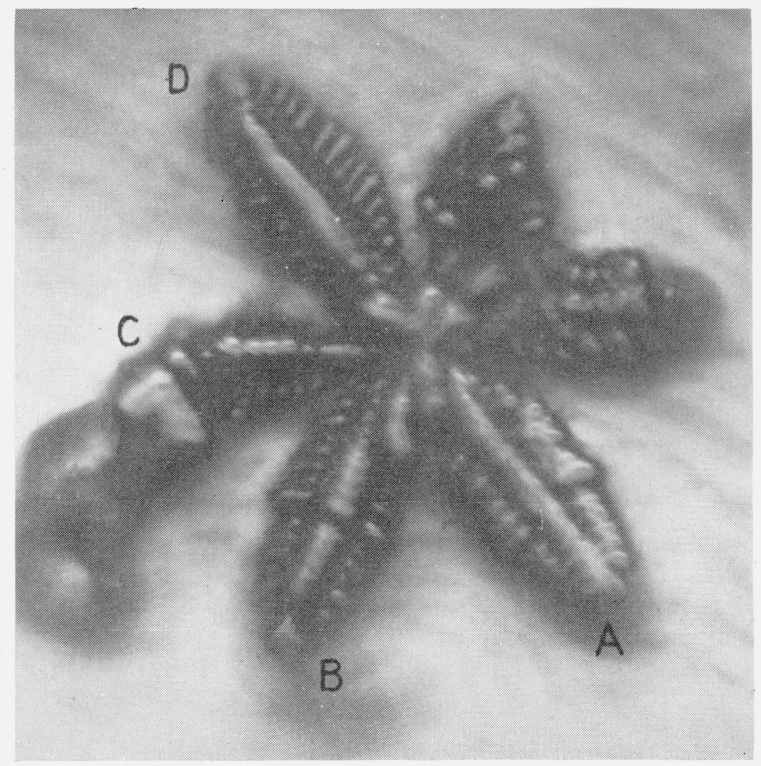

Figure 1. Iron dendrite in magnesium. Etched with glycol reagent. $\times 1,000$.

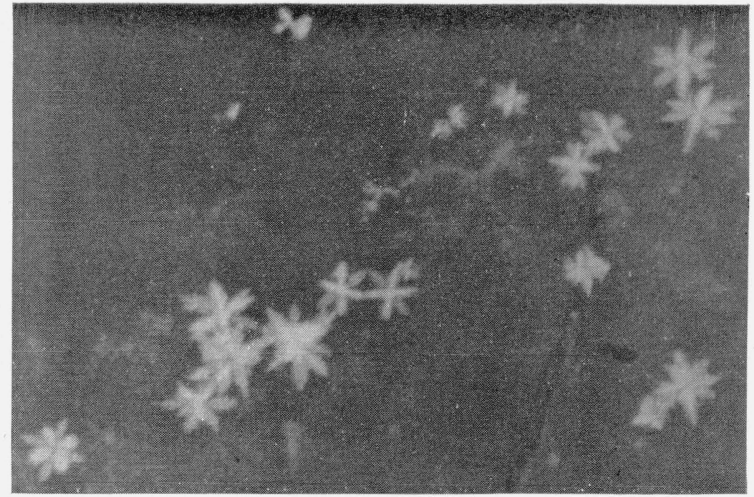

FIgure 2. Photomicroradiograph of a cluster of iron dendrites in magnesium. $\times 250$.

determine if the appearance of certain single dendrites conformed to the appearance of the photographs of the model in the afore-named positions.

The photomicroradiograph of the large dendrite in figure 3 , a is in good agreement with the photograph of the model figure $3, \mathrm{~b}$. In this orientation, all twelve spokes of the model are visible. Six long spokes spaced $60^{\circ}$ apart that are in the plane of the photographic plate, and six spokes that appear shorter due to being inclined $55^{\circ}$. The angular spacing between the twelve spokes when they are projected onto the plane of the photographic plate is $30^{\circ}$. Only three of the inclined spokes are shown on the stereographic projection figure 3, c. The other three are on the opposite side of the projection. Inspection of the photomicroradiograph, figure 3 , a shows that the long branch at the left is not developed completely nor is the short branch at the lower right. However, it is noted that there is a semblance of six long branches and six short branches as shown in figure $3, \mathrm{~b}$, also that the two long branches at the right have minor branches. These minor branches appear to have grown parallel to the long major branches, which should be the case if they grew in dodecahedral directions as shown in figure 3 , c. The relative lengths of the short and long branches compare favorably with the photograph of the model, figure $3, \mathrm{~b}$, which indicates that the dendrite was growing at the same rate in all twelve directions.

In figure 4, a there is a large well-developed dendrite having six long branches. The branches to the left and right are darker than the upper and lower branches. This can be explained by postulating that growth was the same amount in the 

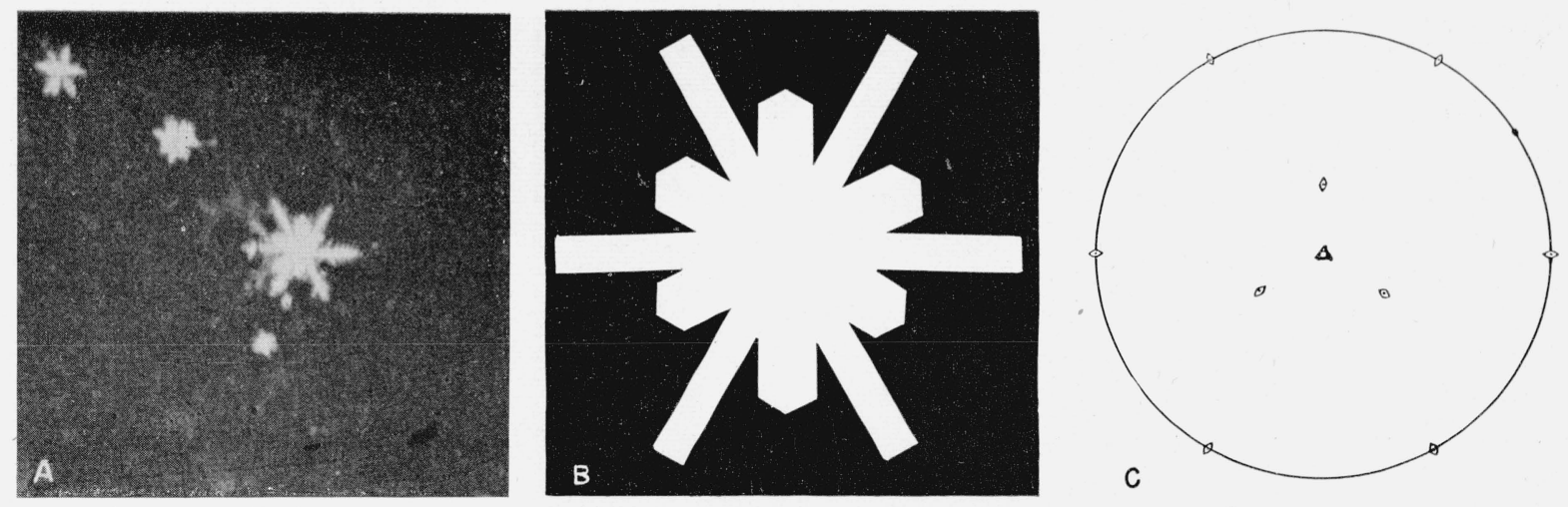

Figure 3. Appearance of an iron dendrite when viewed in octahedral orientation.

A, Photomicroradiograph of iron dendrites in magnesium. x250; B, photograph of a model dendrite that grew at the same rate in the twelve dodecahedral directions; C, stereographic projections showing the orientations of the branches of the iron dendrites and the spokes of the model dendrite.
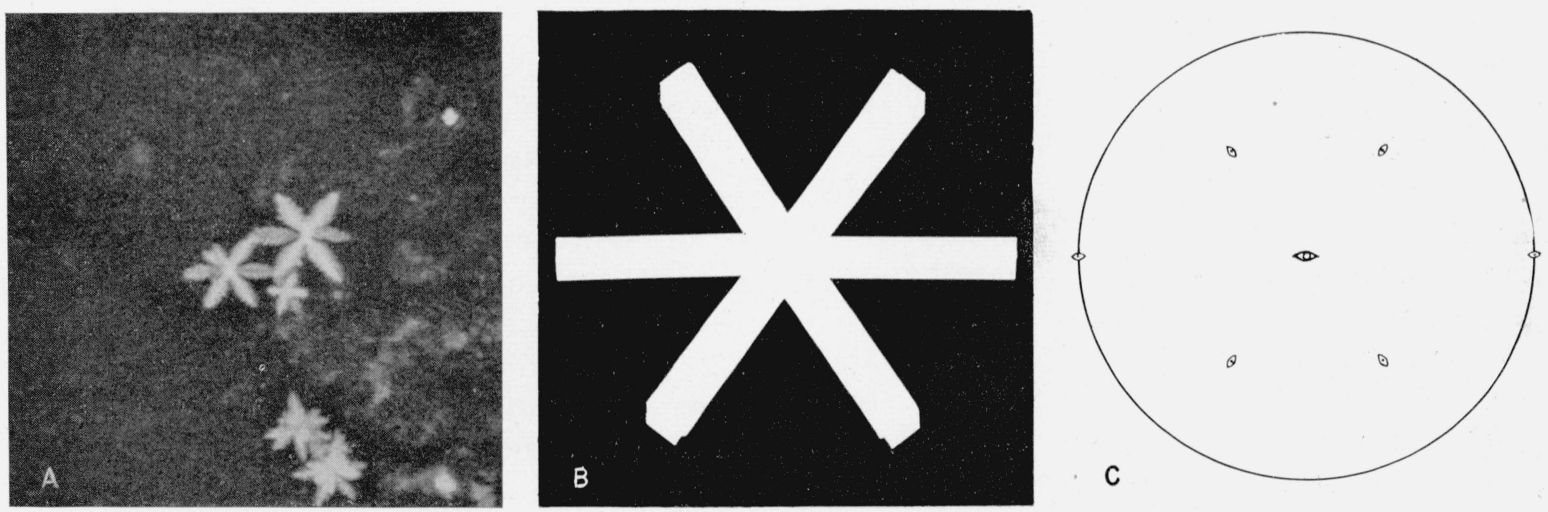

Figure 4. Appearance of an iron dendrite when viewed in dodecahedral orientation.

A, Photomicroradiograph of iron dendrites in magnesium. x250; B, photograph of a model dendrite that grew at the same rate in the twelve dodecahedral directions; $\mathrm{C}$, stereographic projections showing the orientations of the branches of the iron dendrites and the spokes of the model dendrite.
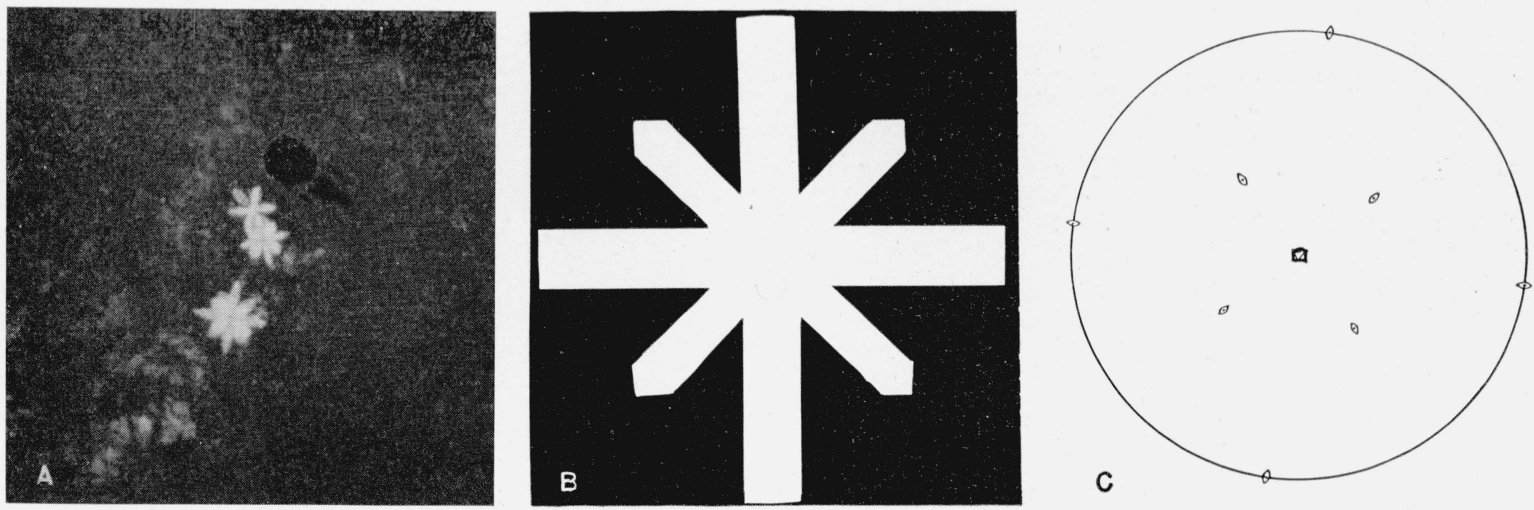

Figure 5. Appearance of an iron dendrite when viewed in cubic orientation.

A, Photomicroradiograph of iron dendrites in magnesium. x250; B, photograph of a model dendrite that grew at the same rate in the twelve dodecahedral directions; C, stereographic projections showing the orientations of the branches of the iron dendrites and the spokes of the model dendrite. 

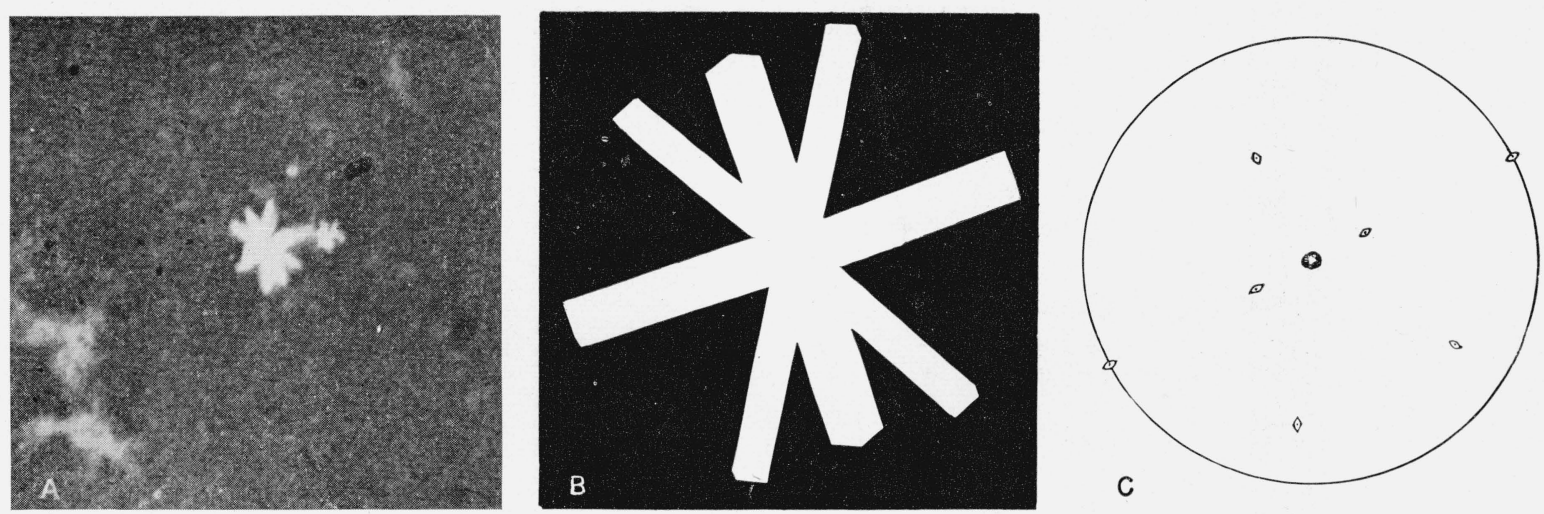

Figure 6. Appearance of an iron dendrite when viewed in icositetrahedral orientation.

A, Photomicroradiograph of iron dendrites in magnesium. x250; B, photograph of a model dendrite that grew at the same rate in the twelve dodecahedral directions; C, stereographic projections showing the orientations of the branches of the iron dendrites and the spokes of the model dendrite.

twelve dodecahedral directions. If the orientation shown in figure 4 , $\mathrm{c}$ is assumed, then there are two branches to the right and left, in the plane of the photograph, eight inclined branches are in pairs, each pair lying in a plane perpendicular to the plane of the photograph, thus the image of each inclined branch is the result of the absorption of X-rays through two branches, whereas in the left and right branches the $\mathrm{X}$-rays have been absorbed in only one kranch. It is noted that the angles between the spokes in figure 4 , b, starting with the one at the left, are $55^{\circ}, 70^{\circ}, 55^{\circ}$, $55^{\circ}, 70^{\circ}$, and $55^{\circ}$. These angles are apparent between the branches in figure 4 , a.

The lower dendrite in figure 5, $\mathrm{A}$ and the one in the lower left corner of figure 2 have four long and four short branches. The four long branches are darker than the four short ones, and the angles between the short and long branches appear to be $45^{\circ}$. The appearance of these dendrites in the photomicroradiographs also can be explained on the basis that growth was in the twelve dodecahedral directions with orientations as shown in figure 5, c. The four long and darker branches are directions in the plane of the photograph, and the four short but lighter branches are pairs of inclined directions in planes perpendicular to the plane of the photograph.

The photomicroradiograph in figure $6, \mathrm{~A}$ is explained on the basis that the dendrite has the orientation indicated in figure $6, \mathrm{~b}$, and 6 , c. The angles between the spokes in figure 6 , b, starting with the one in the lower left corner, are $58^{\circ}, 32^{\circ}$, $32^{\circ}, 58^{\circ}, 58^{\circ}, 32^{\circ}, 32^{\circ}$, and $58^{\circ}$. These appear to agree closely with the angles between the branches in the photomicroradiograph.

As a result of these observations it has been concluded that:

1. Photomicroradiography is a useful tool in determining the number and angular spacing of dendritic arms.

2. Iron dendrites in magnesium grow from a nucleus in the twelve dodecahedral directions.

3. All twelve dendritic arms appear to grow at the same rate.

Washington, November 18, 1949. 